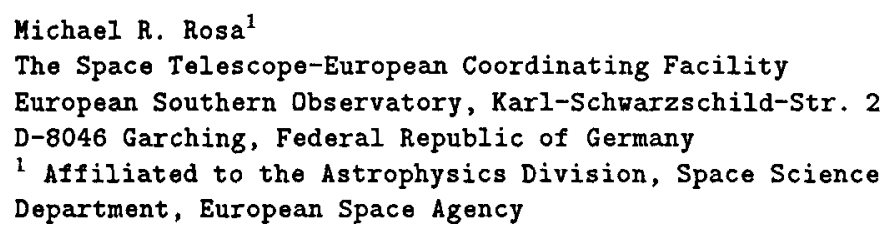

SUMMARY. Experience gained in a world wide effort to exploit restoration methods in order to improve the scientific return from the degraded HST optics calms down fears that HST might have lost all of its spatial resolving power. However, the problems posed by the characteristics of the point spread function (PSF) and of the detectors are such that "black-box" image restoration in an automatic procedure during pipe-line calibration is not applicable. Results from restoration experiments show the directions for future research: On the practical side ways are required to properly estimate the complicated PSF, to objectively describe errors, to treat undersampling and non-linear detectors, and to gain factors in throughput for large amounts of data; innovative example are methods to combine data obtained with differing PSFs (ground - space, space - space) and sampling.

\title{
1. OVERVIEW OF ACTIVITIES
}

Soon after the discovery that HST's science instruments were adversely affected by spherical aberration in the telescope optics, several groups started to experiment with image restoration techniques in order to explore the potentials for improvements in scientific return. First insights pere exchanged at a STSCI workshop in August 1990 (ct. White and Allen,1990), where groups from the STSCI, the ST-ECF, and among others from Caltec, GSFC, JPL, CfA, reported on their work. Further results were discussed at a workshop in Erice in April 1991 (cf. Buccheri etal. 1991). Astrophysical results based on restored HST data from the "First Year of Light" period can be found for example in the two dedicated Astrophysical Journal Letters Vol. 369 No. 2 and Vol. 377 No.1, as well as in the proceedings of a STSCI Conference held in May 1991 (cf. Kinney and Blades 1991).

\section{PSF AND DETECTOR EFFECTS IN HST IMAGING}

Contrary to ground based observations where the atmospheric gaussian like seeing blur suppresses spatial frequencies well before the diffraction limit of the telescopes, the spherically aberrated PSF of HST carries all these frequencies, yet vith greatly diminished contrast with respect to the design goal. The narrow core of the PSF permits near diffraction limit resolution in well exposed raw data, provided sufficient sampling is employed. Additional high spatial frequency information is carried in the diffraction patterns of the supports of the secondary 
and primary mirrors. The very large halo comprising about $90 \%$ of the incident flux acts to reduce both, the faint limit by about $2 \mathrm{mag}$ and the dynamic range. This compromises detection and photometry, particularly in crowded fields.

Equally well contrary to common experience, the PSF's scale is large with respect to the dimensions of the scenes imaged by the detectors. The FOC $f / 288$ PSF covers about one quarter of the field of view. Thus edge effects are very important and pose conceptual problems on restoration procedures: observations are ten incomplete except in the very center of the field, while objects outside the field of view contribute to the intensity distribution inside.

The PSFs are space variant, a particular concern for WFPC data where the use of a given PSF is valid only in a neighborhood with linear dimensions of the order of $10 \%$ of the frame size. The WFPC images further suffer from space variant obscuration of the OTA by the WFPC repeater optics, affecting aperture photometry. The PSFs are furthermore wavelengths dependent, a major concern in broad band filter and white light imaging of sources with unknown energy distributions. Finally, the PSFs are time variant until the outgassing of the HST OTA support structure has leveled off from its initial rate.

By necessity two tracks of PSF estimation have been used: (1) empirical PSFs can be obtained from serendipitous point source observations in the field of view, of which there are usually few if any suitable ones in the small FOC FOVs, or from deliberately scheduled PSF observations. (2) Theoretical PSFs matching empirical PSFs very well in a global sense can be computed from the optical prescriptions (see Burrows, this volume; Krist 1991), but will need to include jitter as well.

Problems for image restoration posed by the characteristics of the detectors are threefold: undersampling, non-linear intensity transfer functions and geometric distortions. Undersampling is in effect for all cameras and modes. The non-linear ITF of the FOC with its limited dynamic range amounts to the fact that a well exposed PSF halo together with a core part still in the linear domain cannot be obtained in the same frame for a given source with a reasonable amount of observing time. The geometric correction in the pipe-line calibration for FOC data causes position dependent noise filtering and correlation.

\section{RESTORATION OF SPECTRAL DATA}

The impacts of the poor HST PSF on the two primary spectrographic facilities of HST are: A severe loss of throughput for the small apertures, a loss of spectral resolution for observations through large apertures, and a loss of spatial purity for observations in crowded fields or of extended sources such as galaxies. The line spread function (LSF) for the smaller apertures retains its original near gaussian shape. For the larger apertures, the convolution of a circular or box shaped profile of dimensions $1-4$ arcsec with the core-halo PSF and subsequent integration onto a $1-D$ array yields LSFs with cores larger by about 50 \& than design and extended broad wings.

The general result of deconvolution experiments on spectral data is the demand for high $S / N$ input data (Gilliland 1990, 1991). Contrary to data of higher dimensions, missing, noisy or damaged data values are much less tolerable. The danger of spurious astrophysical theories being prompted by artefacts simulating narrow spectral lines has to be kept in mind (Lucy 1990). The advice at present is to use small apertures whenever spectral purity is the demand. The signal to noise gained by switching to the larger apertures (from SSA to LSA on the GHRS) 
does notseem to be enough to convincingly recover the SSA results.

\section{RESTORATION METHODS AND FUTURE PROSPECTS}

As briefly outlined above, many factors combine to the success of restoration experiments. The data analysis goal is an important one. In discovery mode restoration prior knowledge and constraints should be kept to a minimum, speed and robustness maximized. Photometric and astrometric properties are of lesser concern. However, the prime mode usually seeks to estimate quantitative information part of which can be drawn from prior knowledge, eg. the non-negativity of intensity. The most successful methods so far seem to be the Richardson-Lucy iterative algorithm (Lucy 1974), and maximum a-posteriori methods such as the maximum entropy methods (Weir and Djorgovski 1991, Gull and Skilling 1991).

Variants to the methods used are currently worked on by several groups in order to improve speed (accelerated deconvolution, Lucy 1991 priv.comm.), to include error estimation, to study the effects of resampling onto finer grids, and the data fusion of images obtained with differing PSFs, $S / N$ and sampling (Lucy and Hook, 1991). The latter method is a promising tool to combine low cost, abundant photons from ground with high spatial resolution information obtained by HST, to become particularly interesting in the post-COSTAR era.

The experiments done sofar demonstrate that given enough signal to noise most of the spatial information spread by the aberrated PSF can be recovered. To be investigated and tested in detail remain the quantitative aspects such as eg. flux conservation, photometric and astrometric fidelity. The spectral domain has, unfortunately, been left aside sofar in large scale experiments on restoration. Because of the simplicity of LSFs with respect to the 2D HST PSFs, spectral deconvolution certainly deserves more interest in future. Roads of development in $2 \mathrm{D}$ and 1D restoration are methods making full use of a priory knowledge. Optical astronomy only in 1990 entered the restoration era, a field under exploration in radio astronomy since decades. Much has yet to be done, experimenting and campaigning, in order to make image restoration a widely accepted part in the tool box of optical astronomy.

\section{REFERENCES}

Buccheri R. etal, 1991, "4th Int. Workshop on Data Analysis in Astronomy", Erice April 1991, Plenum Press

Gilliland R.L., 1990, in (White and Allen, 1990 cit below), 7

Gilliland R.L., 1991, in 'SSTSCI Newsletter', Vol 8, No. 2, 8

Gull S.F., Skilling J., 1991, 'MemSys5 User's Manual'', M.E.Consultants Ltd

Lucy L.B., 1974, AJ 79,745

Lucy L.B., 1990, in (White and Allen, 1990 cit below), 80

Lucy L.B., Hook R., 1991, in '1st Ann. Conf. on Astronomical Data Analysis Systems and Software' ', Tucson Nov. 1991, in press

Kinney A.L., Blades J.C., 1991: "The First Year of HST Observations', Proceedings of STScI Workshop, May 1991, STScI, Baltimore, 1991

Krist J., 1991, “The TIM Cookbook', STScI Internal Report, Feb. 1991

Weir N., Djorgovski S., 1991, in (White and Allen 1991, cit. below), 31

White R.L., Allen R.J., 1990: 'The Restoration of HST Images and Spectra', Proceedings of STScI Workshop, August 1990, STScI, Baltimore, 1991 